\title{
Prevalencia de valores inadecuados de vitamina D y factores de riesgo asociados en jóvenes universitarios de Asunción
}

\author{
Acosta Colman Isabel ${ }^{1}$, Martínez María Teresa ${ }^{2}$, Sanabria Diego, Yinde Yanira ${ }^{1}$, Colmán Nelly ${ }^{1}$, Ojeda \\ Aldo $^{1}$, Román Lourdes ${ }^{1}$, Losanto Jonathan ${ }^{1}$, $\operatorname{Vazquez}^{\operatorname{Marcos}^{1}}{ }^{1}$, Duarte Margarita ${ }^{1}$ \\ ${ }^{1}$ Universidad Nacional de Asunción, Facultad de Ciencias Médicas, Hospital de Clínicas, \\ Departamento de Reumatología. San Lorenzo, Paraguay \\ ${ }^{2}$ Laboratorios Curie. Paraguay
}

Cómo referenciar este artículo/ How to reference this article:
Acosta Colman I, Martínez MT, Sanabria D, Yinde Y, Colmán N, Ojeda A, et al. Prevalencia de valores inadecuados de vitamina $D$ y factores de riesgo asociados en jóvenes universitarios de la ciudad de Asunción. Mem. Inst. Investig. Cienc. Salud. 2019; $17(2): 36-43$

\section{R E S U M E N}

La vitamina $D$ es una hormona cumple una función en la regulación de numerosos genes que participan en la proliferación y maduración celular. El objetivo de este trabajo fue determinar la prevalencia de valores inadecuados de esta vitamina en jóvenes sanos de Asunción e identificar los posibles factores de riesgo asociados. Estudio observacional descriptivo basado en datos clínico-epidemiológicos y en la determinación de la 25hidroxivitamina $\mathrm{D}(25-\mathrm{OH}$ vitamina $\mathrm{D})$ por el método de quimioluminiscencia (CMIA). Se consideró valor adecuado $\geq 30 \mathrm{ng} / \mathrm{dl}$, inadecuado por debajo de este rango y deficiente $\leq 20$ $\mathrm{ng} / \mathrm{dl}$. El análisis estadístico se realizó con el programa R-proyect. Para establecer factores de riesgo para tener deficiencia de vitamina $D$ se utilizó regresión logística bi y multivariante. Se incluyeron 817 jóvenes universitarios de edad promedio 21 años. La concentración de vitamina $D$ tuvo una media de $25 \pm 8 \mathrm{ng} / \mathrm{dl}$, oscilando entre 64,3 y 2,4 $\mathrm{ng} / \mathrm{dl}$. El $75,5 \%$ de los participantes tenían valores inadecuados de vitamina $\mathrm{D}$. La deficiencia de vitamina $D$ fue significativamente $(p=0,006)$ más frecuente en mujeres, personas con sobrepeso $(p=0,03)$, con falta de exposición al sol $(p=0,001)$ y con sedentarismo $(p=0,0001)$. Este estudio confirma la elevada prevalencia de valores inadecuados de vitamina $D$ en nuestro país y permite definir un perfil de muy alto riesgo para las jóvenes sedentarias y con sobrepeso, lo que justifica una atención especial de parte de los médicos y las instituciones responsables de la salud pública en nuestro país.

Palabras clave: Vitamina $D$, valores inadecuados, jóvenes universitarios sanos.

\section{Prevalence of inadequate vitamin D values and associated risk factors in university students of the city of Asunción}

\section{A B S T R A C T}

Vitamin $D$ is a hormone that plays a role in the regulation of numerous genes involved in cell proliferation and maturation. The objective of this work was to determine the prevalence of inadequate values of this vitamin in healthy young people of the city of Asunción and to identify the possible risk factors associated with it. Descriptive observational study based on clinical-epidemiological data and in the determination of 25hydroxyvitamin $\mathrm{D}(25-\mathrm{OH}$ vitamin $\mathrm{D})$ by the chemiluminescence method (CMIA). It was considered appropriate values $\geq 30 \mathrm{ng} / \mathrm{dl}$, inappropriate below this range and deficient $<20$

Fecha de recepción: junio 2019. Fecha de aceptación: julio 2019

*Autor correspondiente: Margarita Duarte. Departamento de Reumatología, Hospital de Clínicas de San Lorenzo, Asunción, Paraguay

Email: duartemussi@gmail.com 
$\mathrm{ng} / \mathrm{dl}$. Statistical analyzes were carried out with the R-project program. To establish the association, bivariate and multivariate logistic regression was used. We included 817 university students with an average age of 21 years. The average value of the vitamin $D$ concentration was $25 \pm 8 \mathrm{ng} / \mathrm{dl}$, ranging from 64.3 to $2.4 \mathrm{ng} / \mathrm{dl}$. Inadequate vitamin $D$ values were found in $75.5 \%$ of the participants. Vitamin D deficiency was significantly ( $p$ $<0.006)$ more frequent in women, overweight people $(p<0.03)$, lack of sun exposure $(p=$ 0.001 ) and sedentary lifestyle $(p=0.0001)$. This study confirms the high prevalence of inadequate vitamin $D$ values in our country and allows us to define a very high risk profile for sedentary and overweight young women, which justifies special attention from physicians and institutions responsible for public health in our country.

Keywords: Vitamin D, inadequate values, healthy university students.

\section{INTRODUCCIÓN}

La vitamina $D$, o mejor llamada, la hormona $D$, desempeña un rol fundamental en la absorción del calcio en el intestino delgado y en el desarrollo y el mantenimiento del tejido óseo a lo largo de toda la vida. La carencia de la vitamina $\mathrm{D}$ es la causa del raquitismo en los niños y de la osteomalacia en los adultos, enfermedades asociadas a dolor y deformidades óseas que limitan el movimiento y predisponen a fracturas del esqueleto ${ }^{(1)}$.

La cantidad de vitamina $D$ en nuestro organismo depende principalmente de la exposición a la luz solar. Otros factores que tienen trascendencia en la producción y la biodisponibilidad de esta hormona son el color de la piel, el sexo, la cantidad de tejido graso de nuestro cuerpo y la actividad física realizada(2). No se sabe con certeza si la actividad física representa un factor independiente o si su efecto es debido a la mayor probabilidad de exposición al sol de los que hacen ejercicios físicos, así como la distribución diferente de los tejidos graso y muscular en estos sujetos ${ }^{(3)}$.

En las dos últimas décadas ha habido un renacimiento del interés en esta hormona, como consecuencia del descubrimiento de nuevas funciones que no están relacionadas al metabolismo fosfocálcico. Estudios in vitro han demostrado que la vitamina $D$ actúa sobre numerosos genes que regulan la proliferación celular, diferenciación celular, la respuesta inmune a las infecciones, la ateromatosis y las secreciones de hormonas como la insulina ${ }^{(2,4,5)}$.

La concentración óptima de vitamina $D$ para mantener una adecuada salud ósea puede no ser la misma que la necesaria para sus acciones extraóseas, y existe un gran debate sobre los valores recomendados para cada una de estas situaciones ${ }^{(6-8)}$ Este debate se traslada también a la nomenclatura relacionada a los valores ideales, adecuados, suficientes o deficientes del 25 hidoxicolecalciferol, que por el momento, se refieren en particular al efecto de la vitamina $D$ sobre el metabolismo del calcio y del tejido óseo.

Paralelamente a estos desafiantes descubrimientos en relación a las funciones de la vitamina $D$, se ha constatado que una gran parte de la población mundial tiene niveles de vitamina $D$ muy por debajo de aquellos considerados adecuados, incluso en zonas con abundante luz solar durante gran parte del año, como en Centroamérica y Australia ${ }^{(9-13)}$.

En nuestro país, dos estudios realizados con pequeño número de pacientes encontraron una alta prevalencia de valores deficientes e insuficientes de vitamina $\mathrm{D}^{(14,15)}$.

Considerando las posibles implicancias relacionadas tanto a la salud ósea como a sus funciones en tejidos extraóseos, hemos realizado un estudio en jóvenes universitarios de distintas carreras que residen en la ciudad de Asunción, con el objeto de determinar la prevalencia de valores inadecuados de vitamina $D$ y sus posibles asociaciones con factores antropométricos, hábitos alimentarios y estilos de vida en nuestra población.

\section{METODOLOGÍA}

Se realizó un estudio observacional descriptivo de corte transversal, con un componente analítico, con el objetivo de determinar la prevalencia de valores inadecuados de vitamina D y los factores de riesgos asociados a la misma en jóvenes sanos.

Se incluyeron jóvenes estudiantes de diferentes carreras universitarias que residen en Asunción y Gran Asunción, quienes fueron evaluados por médicos del Departamento de Reumatología del Hospital de Clínicas entre los meses de marzo del año 2015 y noviembre del año de 2017. Se registraron los datos y antecedentes personales y familiares, y se llevó a cabo un interrogatorio dirigido y detallado sobre los hábitos nutricionales, la actividad física y la exposición al sol de cada participante. 
Se determinaron los niveles de 25-hidroxivitamina $D$ (25-OH vitamina $D$ ) empleando la prueba de inmunoensayo de micropartículas por quimioluminiscencia (CMIA), disponible comercialmente con el nombre de ARCHITECT 25-OH Vitamina D (Abbott). Para los fines de este estudio, consideramos valores adecuados a aquellos iguales o mayores a $30 \mathrm{ng} / \mathrm{dl}$, e inadecuados los que estén por debajo de este rango. Entre los individuos con valores inadecuados, hemos considerado además el subgrupo de deficiencia de vitamina $D$, que incluye a aquellos con valores iguales o menores a $20 \mathrm{ng} / \mathrm{dl}$.

Entre las variables analizadas figuran: edad, sexo, raza, color de piel, índice de masa corporal (IMC), ingesta de lácteos, huevos y pescado, actividad física regular (al menos dos veces por semana por más de 30 minutos), exposición solar, uso de bloqueadores solares y estación del año en el cual fue tomada la muestra de sangre.

Los datos fueron ingresados en una planilla Microsoft Excel y los análisis estadísticos se realizaron con el programa R-proyect. Para la descripción de las características de los participantes, las variables cualitativas fueron expresadas como porcentajes y las cuantitativas de acuerdo a su media y desvío estándar. En cuanto al análisis de asociación entre las variables consideradas y los niveles de vitamina $D$, se calculó el odds ratio (OR) para la deficiencia de la vitamina $D$ y se comparó con niveles adecuados de la misma, estimado mediante el ajuste de un modelo de regresión logística univariada. Los resultados fueron expresados en intervalos de confianza estimados al $95 \%$ de confiabilidad.

Las asociaciones significativas fueron consideradas aquellas cuyo valor de $\mathrm{p}$ fue menor o igual a 0,05 utilizando el test del chi2.

Para determinar los factores de riesgos fue utilizado un modelo de regresión logística multivariante de tal forma a estimar las probabilidades de tener niveles deficientes de vitamina $D$, con el fin de obtener perfiles de menor y mayor riesgo para el estado de deficiencia de vitamina $D$.

\section{RESULTADOS}

Fueron incluidos 817 jóvenes universitarios de la ciudad de Asunción, con edades comprendidas entre los 17 y 43 años, con un promedio de 21 años de edad. El 69,1\% de los participantes fueron del sexo femenino y 84,7 de raza hispana. En el $75,7 \%$ de los casos el color de la piel era marrón claro o moreno y el índice de masa corporal fue menor de 25 en $62,3 \%$ de los jóvenes evaluados (Tabla 1 ).

Tabla 1: Distribución por sexo y color de piel de jóvenes universitarios de la ciudad de Asunción

\begin{tabular}{llrr}
\hline & Variables & Recuento & Porcentaje \\
\hline Sexo & Mujer & 564 & $69,1 \%$ \\
& Hombre & 252 & $30,9 \%$ \\
\multirow{3}{*}{ Raza } & Total & $\mathbf{8 1 6}$ & $\mathbf{1 0 0 , 0 \%}$ \\
& Hispana & 686 & $84,7 \%$ \\
\multirow{4}{*}{ Color de piel } & Caucásica & 124 & $15,3 \%$ \\
& Total & $\mathbf{8 1 0}$ & $\mathbf{1 0 0 , 0 \%}$ \\
& Moreno o trigueño & 618 & $75,7 \%$ \\
& Blanco & 198 & $24,3 \%$ \\
& Total & $\mathbf{8 1 6}$ & $\mathbf{1 0 0 , 0 \%}$ \\
\hline
\end{tabular}

En relación a los hábitos alimentarios, el 90 \% de los jóvenes refirió consumir una taza o más de leche cada día y 1 o más huevos cada semana. El 48,3\% afirmó comer pescado, con una frecuencia de al menos una vez al mes. La práctica de ejercicios regulares fue habitual en $52,4 \%$ de los estudiantes, y el $52,5 \%$ de ellos se expone al sol más de 15 minutos cada día. $28,4 \%$ refirió una exposición solar de más 30 minutos cada día. En relación al uso de protectores solares, el $17,5 \%$ de los participantes refirió usar protector solar, pero sólo en la cara (Tabla 2). 
Tabla 2: Distribución según dieta, ejercicios, exposición solar e IMC de jóvenes universitarios de la ciudad de Asunción.

\begin{tabular}{|c|c|c|c|}
\hline Variables & & Recuento & Porcentaje \\
\hline Consumo de leche & No consume & 70 & $8,6 \%$ \\
\hline & Una taza & 405 & $49,6 \%$ \\
\hline & Dos o más tazas & 342 & $41,9 \%$ \\
\hline & Total & 817 & $100,0 \%$ \\
\hline Consumo de pescado & $\mathrm{Si}$ & 392 & $48,3 \%$ \\
\hline & No & 420 & $51,7 \%$ \\
\hline & Total & 812 & $100,0 \%$ \\
\hline Consumo de huevo & No & 85 & $10,5 \%$ \\
\hline & $1 \circ 2$ & 325 & $40,3 \%$ \\
\hline & 3 o más & 396 & $49,1 \%$ \\
\hline & Total & 806 & $100,0 \%$ \\
\hline Ejercicio & $\mathrm{Si}$ & 384 & $47,6 \%$ \\
\hline & No & 423 & $52,4 \%$ \\
\hline & Total & 807 & $100,0 \%$ \\
\hline Exposición al sol & No & 161 & $19,8 \%$ \\
\hline & 15 o menos & 226 & $27,8 \%$ \\
\hline & Entre 16 y $30 \mathrm{~min}$ & 196 & $24,1 \%$ \\
\hline & Más de $30 \mathrm{~min}$ & 231 & $28,4 \%$ \\
\hline & Total & 814 & $100,0 \%$ \\
\hline Uso de protector solar & $\mathrm{Si}$ & 141 & $17,5 \%$ \\
\hline & No & 667 & $82,5 \%$ \\
\hline & Total & 808 & $100,0 \%$ \\
\hline Nivel de IMC & Menor a 25 & 498 & $62,3 \%$ \\
\hline & 25 o más & 302 & $37,8 \%$ \\
\hline & Total & 800 & $100,0 \%$ \\
\hline
\end{tabular}

El valor de la media de la concentración de vitamina $D$ en la población estudiada fue de $25 \pm 8 \mathrm{ng} / \mathrm{dl}$ oscilando entre 64,3 y $2,4 \mathrm{ng} / \mathrm{dl}$. El 75,5\% de los jóvenes tenían valores inadecuados de vitamina $D$, es decir, de menos de $30 \mathrm{ng} / \mathrm{dl}$. Entre las mujeres, 32,1\% tenía valores iguales o menores a $20 \mathrm{ng} / \mathrm{dl}$, y entre los varones el 23,8\%, lo que corresponde a valores deficientes de vitamina D (Figura 1 ).

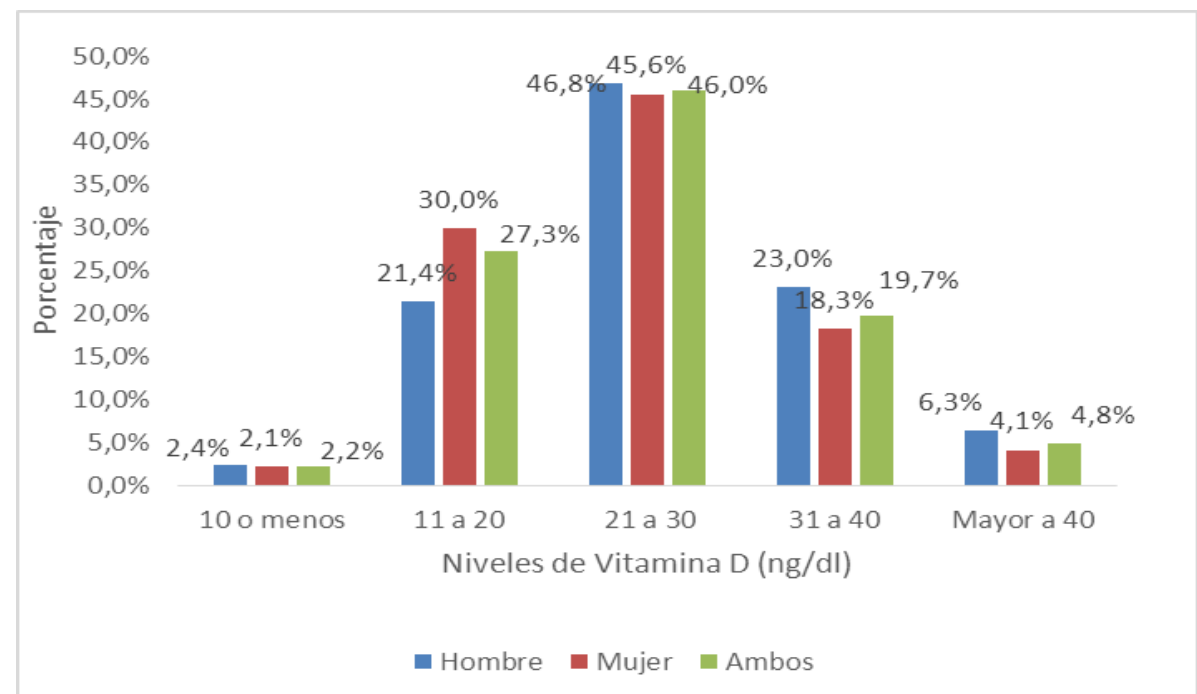

Figura 1: Valor de la media de la concentración de vitamina $D$ en jóvenes universitarios de la ciudad de Asunción.

En la Figura 2 se muestran las variaciones estacionales de los niveles de vitamina $D$, siendo la concentración media $3,2 \mathrm{ng} / \mathrm{dl}$ menor en los meses de poco sol (otoño e invierno) comparada con los meses de mucho sol (primavera y verano). 


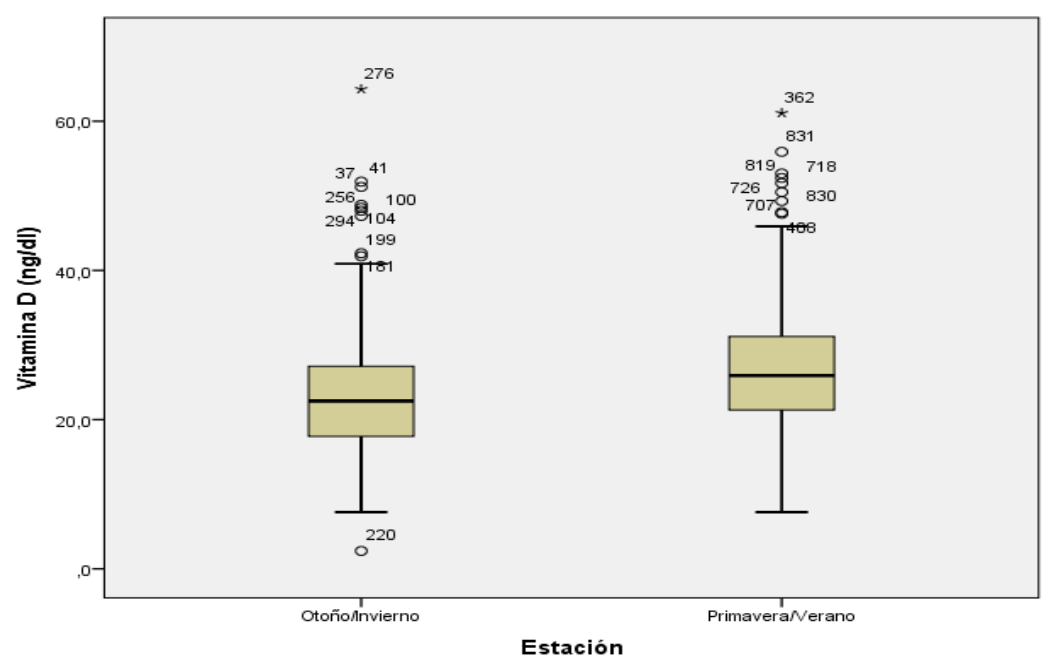

Figura 2: Variaciones estacionales de los niveles de vitamina $D$ en jóvenes universitarios de la ciudad de Asunción.

Comparando los individuos con deficiencia de vitamina $D$ con aquellos con valores adecuados de vitamina $D$, no se encontraron diferencias significativas asociadas a la raza, el color de piel, los hábitos nutricionales ni a la utilización de protectores solares.

En relación al sexo, la deficiencia de vitamina $D$ fue significativamente más frecuente en mujeres que en varones $(p=0,006)$, así como en personas con sobrepeso $(p=0,03)$. La falta de exposición al sol y el sedentarismo también se asociaron significativamente a la deficiencia de vitamina $D(p=0,001$ y $p=0,0001$ respectivamente). Tanto los individuos que no se exponían al sol como los que se exponían 15 minutos diarios o menos tenían mayor riesgo, comparados con los que se exponían 16 a 30 minutos y más de 30 minutos respectivamente.

En la Tabla 3 se presentan los cálculos de probabilidad de padecer déficit de vitamina $D$ en relación a las variables estudiadas. Los cálculos de probabilidad basados en estos datos muestran que un $90 \%$ de los estudiantes de sexo femenino que tienen sobrepeso, que no hacen ejercicios ni se exponen al sol padecerán deficiencia de vitamina $D$ en los meses de otoño e invierno. En los meses de verano, esta probabilidad es del $70 \%$. Por otro lado, la probabilidad de padecer deficiencia de vitamina $D$ para un estudiante universitario de sexo masculino, con índice de masa corporal menor de 25, que hace ejercicios y se expone al sol regularmente es de $17 \%$ en los meses de verano y de $40 \%$ en los meses de otoño o invierno.

Tabla 3: Probabilidad de padecer déficit de vitamina $D$ en relación a las variables estudiadas.

\begin{tabular}{lll}
\hline Variables & Bajo Riego & Alto Riesgo \\
\hline Sexo & Hombre & Mujer \\
Estación & Sol (Verano/ Primavera) & Menos sol (Invierno/Otoño) \\
Ejercicio Regular & Si & No \\
Exposición al sol & Más de 30 minutos & No \\
IMC & menor de 25 & mayor o igual de 25 \\
Probabilidad & $\mathbf{1 7 \%}$ & $\mathbf{9 0 \%}$ \\
\hline
\end{tabular}

\section{DISCUSIÓN}

El presente estudio fue realizado en jóvenes sanos residentes en Asunción y permitió determinar la prevalencia de valores inadecuados de vitamina $D$ en este grupo etario y las asociaciones entre los niveles de vitamina D y las características antropométricas, geográficas y nutricionales de los participantes.

Hemos constatado que el $75 \%$ de los jóvenes universitarios residentes en Asunción tienen niveles inadecuados de vitamina D. Este estudio constituye el primer estudio 
poblacional realizado en Paraguay sobre la presencia de valores inadecuados de vitamina $D$ y de deficiencia de la misma.

Numerosos estudios provenientes de Sudamérica, el Caribe, África, Asia y Australia han encontrado valores inadecuados de vitamina $D$ hasta en $90 \%$ de los pacientes ${ }^{(10-13)}$ por lo que nuestros resultados están en concordancia con estos hallazgos y destacan la importancia de considerar esta situación desde el punto de vista de sus implicancias en salud pública.

La presencia de melanina, así como el uso de protectores solares disminuye considerablemente la cantidad de luz ultravioleta que llega a las capas profundas de la piel $^{(16,19)}$. Los individuos de piel oscura sintetizan menos vitamina $D$ que los de pieles claras en condiciones similares de exposición solar. No obstante, en este estudio no hemos encontrado diferencias en los niveles de vitamina $D$ entre los participantes de origen hispano y los caucásicos, ni tampoco en relación al color de la piel. Una posible explicación es que en nuestro país existe una importante población mestiza, con ancestros tanto caucásicos como hispanos, lo que puede dificultar hallar asociaciones relacionadas a estas variables.

Hemos constatado que las mujeres presentaban niveles más bajos de vitamina $D$ que los varones. Si bien algunos estudios han encontrado una mayor prevalencia de valores inadecuados de vitamina $D$ en varones ${ }^{(20)}$, otros reportan mayor prevalencia en mujeres $^{(11,13,21)}$. En éstas, el mayor porcentaje de tejido graso en la composición corporal total y las hormonas sexuales femeninas tienen efectos particulares en la biodisponibilidad de la vitamina D. Existe una relación inversa entre el índice de masa corporal (IMC) y los niveles de Vitamina $D$ en plasma. La dinámica de la interrelación entre los depósitos tisulares de vitamina $D$ y los niveles en circulación no se conocen en detalle, pero parece plausible que exista una dilución volumétrica de la vitamina $D$ en el tejido graso, y que por este motivo se necesite un mayor aporte de vitamina $D$ para saturar el tejido adiposo ${ }^{(22,23)}$. Esta situación puede relacionarse también a la menor exposición al sol y la menor actividad física de los pacientes con sobrepeso y obesidad, lo que condicionaría un déficit aún mayor de vitamina $D$ en los individuos de sexo femenino.

Nuestros resultados muestran que las mujeres con sobrepeso, vida sedentaria y poca exposición al sol tienen un $90 \%$ de probabilidad de tener valores de vitamina $D$ por debajo de $20 \mathrm{ng} / \mathrm{dl}$ durante los meses de invierno y el riesgo sigue siendo elevado en el verano. Este es un grupo fácilmente identificable de la población general que puede beneficiarse con medidas preventivas adecuadas para disminuir el riesgo de tener valores inadecuados de vitamina D.

Si bien en el sexo masculino, el peso adecuado, la exposición al sol y la actividad física regular pueden considerarse factores relacionados positivamente con valores adecuados de vitamina $D$, hemos encontrado que la probabilidad de este subgrupo de padecer deficiencia de vitamina $D$ en meses de otoño e invierno es de $40 \%$. Es decir, cerca de la mitad de los jóvenes que no tienen factores de riesgo también pueden ser deficientes de vitamina $D$ en nuestro país.

No hemos encontrado diferencias asociadas a los hábitos alimentarios en este estudio. La ingesta de pescado no es habitual ni frecuente en nuestra población, y si bien los lácteos y huevos son parte fundamental de la dieta, no se relacionan con niveles adecuados de vitamina $D$. Este resultado permite inferir que la suplementación de la vitamina $D$ en los lácteos disponibles en nuestro país no es suficiente para mejorar el estatus de esta vitamina en la población joven, y aumentar la cantidad suplementada puede ser una estrategia preventiva sencilla y económica.

Si bien este estudio incluyó solamente jóvenes de nivel universitario de la ciudad de Asunción, los resultados podrían ser transpolables a la mayor parte de la población joven universitaria del país, ya que las variables antropométricas y geográficas son similares en gran parte de nuestro territorio. Sin embargo, las variables relacionadas al estado socioeconómico, como la alimentación y el tiempo dedicado al ejercicio, pueden ser diferentes en aquellos jóvenes que no tienen acceso a la educación terciaria, por lo que se necesitan más estudios para conocer a fondo la prevalencia de valores inadecuados de vitamina $\mathrm{D}$ en otros grupos de la población de nuestro país. 
Consideramos que nuestros resultados demuestran plenamente que, en nuestro país, así como en gran parte de los países del mundo, la situación de los niveles bajos de vitamina D debe ser apreciada como un problema de salud pública que requiere mayor atención tanto de los organismos gubernamentales abocados a la prevención primaria como de los colegas que asisten actualmente a pacientes con osteoporosis y fracturas.

Financiación: Este estudio fue realizado dentro del marco del Proyecto 14-INV-467 "Proyecto Piloto: Vitamina D y otros parámetros del metabolismo fosfo-cálcico en individuos sanos universitarios de Asunción apoyado por PROCIENCIA/CONACYT, Paraguay, convocatoria 2013.

\section{REFERENCIAS BIBLIOGRÁFICAS}

1. DeLuca HF.Overview of general physiologic features and functions of vitamin $D$, The American Journal of Clinical Nutrition.2004;80(6)1689-1696

2. Holick MF. Vitamin D deficiency. N Engl J Med 2007;357:266-81

3. Constantini NW, Dubnov-Raz G, Chodick G, Rozen GS, Giladi A, Ish-Shalom S. Physical activity and bone mineral density in adolescents with vitamin D deficiency. Med Sci Sports Exerc. 2010;42(4):646-50.

4. Dobnig $\mathrm{H}$, Pilz $\mathrm{S}$, Scharnagl $\mathrm{H}$. Independent association of low serum 25hydroxyvitamin D and 1,25dihydroxyvitamin $D$ levels with all-cause and cardiovascular mortality. Arch Intern Med 2008; 168:1340-9.

5. Hewison M. Vitamin D and innate immunity. Curr Opin Investig Drugs 2008; 9: 485-90.

6. Lips P. Which circulating level of 25hydroxyvitamin D is appropriate? J Steroid Biochem Mol Biol. 2004;89-90(1-5):6114.

7. Holick MF. Sunlight and vitamin D for bone health and prevention of autoimmune diseases, cancers, and cardiovascular disease. Am J Clin Nutr. 2004;80(6 Suppl):1678S-88.

8. Rosen C. Vitamin D Insufficiency. N Engl J Med 2011;364:248-54.

9. McKenna M, Freaney R. Secondary Hyperparathyroidism in the Elderly: Means to Defining Hypovitaminosis D. Osteoporosis International.1998;8(5):3-6

10. Forrest $K$, Stuhldreher $W$. Prevalence and correlates of vitamin $D$ deficiency in US adults. Nutrition Research. 2011(31):4854.

11. Hyppönen E, Power C. Hypovitaminosis D in British adults at age $45 \mathrm{y}$ : nationwide cohort study of dietary and lifestyle predictors. The American Journal of Clinical Nutrition.2007;85(3): 860-8.

11. Brito A, Cori H, Olivares M, Mujica FM, Cediel G, López de Romaña D. Less than adequate vitamin $\mathrm{D}$ satus and intake in Latin America and the Caribbean: a problem of unknown magnitude. Food Nutr Bull 2013;34(1):52-64.

12. Lips $P$, Hosking D, Lippuner K, Norquist JM, Wehren L, Maalouf G, Ragi.Eis S, Chandler J. The prevalence of vitamin $D$ inadequacy amongst women with osteoporosis: an international epidemiological investigation. J Intern Med. 2006; 260(3):245-54.

13. Daly R, Gagnon C, Lu Z, Magliano $D$, Dunstan D , Sikaris K , Zimmet $P$ et al. Prevalence of vitamin $D$ deficiency and its determinants in Australian adults aged 25 years and older: a national, populationbased study. Clinical Endocrinoly.2012; 77(1):26-35.

14. Aquino $A$, Ojeda $A$, Colman $N$, Yinde $Y$, Acosta ME, Acosta Colmán I, Duarte M. Vitamin $D$ deficiency in post-menopausal patients and its relation with mineral metabolism and osteoporosis. Mem. Inst. Investig. Cienc. Salud, Vol. 11(1) Junio 2013: 39-44

15. Ojeda A, Duarte M, Echeverría E, Meyer MT, Duarte N, de Pratt $T$, Samudio $M$. Frequency of Vitamin $D$ insufficiency in healthy young adults of Asunción. Mem. Inst. Investig. Cienc. Salud, Vol. 12(1) junio 2014: 26-32.

16. Matsuoka LY, Wortsman J, Haddad JG, Kolm P, Hollis BW. Racial pigmentation and the cutaneous synthesis of vitamin D. Arch Dermatol.1991; 127:536-8.

17. Bogaczewicz J, Karczmarewicz E, Pludowski P, Zabek J, Wozniacka A. Requirement for vitamin $D$ supplementation in patients using photoprotection: variations in vitamin $D$ levels and bone formation markers. International Journal of Dermatology.2016;55(4):176-183.

18. Ginde $A A$, Liu MC, Camargo CA Jr. Demographic differences and trends of vitamin $D$ insufficiency in the US population, 1988-2004. Arch Intern Med. 2009; 169(6):626-32.

19. Powe CE, Evans MK, Wenger J, Zonderman $A B$, Berg $A H$, Nalls $M$, et al. Vitamin Dbinding protein and vitamin $D$ status of black Americans and white Americans. N Engl Med 2013; 369:1991-2000

20. Tønnesen R, HambakHovind P, Thorbjørn Jensen $L$, Schwarz P. Determinants of vitamin $D$ status in young adults: influence of lifestyle, sociodemographic and anthropometric factors. BMC Public Health (2016) 16:385.

21. Yao Y, Shihui F, Li N, Hu F, Zhang H, Zhu $\mathrm{Q}$ et al. Sex, residence and fish intake predict vitamin $D$ status in Chinese 
Centenarians. The Journal of nutrition, health and aging.2019;23(2):165-171.

22. Censani $M$, Stein $E$, Shane $E$,Oberfield $S$, McMahon D, Lerner $S$ et al.Vitamin $D$ deficiency is prevalent in morbidly obese adolescents prior to bariatric surgery. Hindawi Publishing Corporation.2013:1-7

23. Samuel $L$, Borrell LN. The effect of body mass index on adequacy of serum 25hydroxyvitamin D levels in US adults: the National Health and Nutrition Examination Survey 2001 to 2006. Ann Epidemiol. $2014 ; 24(10): 781-4$. 